\title{
Hadronic Production of Heavy Quarks
}

\author{
Matteo Cacciari ${ }^{2}$ \\ Deutsches Elektronen-Synchrotron DESY \\ D-22603 Hamburg, Germany
}

\begin{abstract}
We review the status of theoretical evaluations of heavy quark and heavy quarkonium hadroproduction cross sections and their comparisons with experimental data.
\end{abstract}

\section{INTRODUCTION}

When, more than twenty years ago, charm was discovered [1] and subsequently interpreted [2] as the first heavy quark ever observed, it came as a big surprise. The discovery of bottom [3] a few years later produced perhaps (I wasn't around at that time...) less excitement, but was surely equally important in extending our knowledge of what is now called the heavy quark sector.

This sector is today well known, and probably recently completed by the discovery of the top quark [4]. Theoretical physics of heavy quarks has therefore shifted gear, and moved from "discovery mode" to "precision physics."

The new name of the game is now testing Quantum Chromodynamics, by checking its predictions against experimental results. The latter have now grown quite accurate, and therefore demand equally precise theoretical calculations. In this talk I shall describe the state of the art of such calculations for heavy quark hadroproduction.

I shall first review the fixed-order next-to-leading order (NLO) QCD calculation, now available for total cross sections and one- and two-particles distributions. It is a consolidated result and provides a benchmark for future developments.

Large logarithms appear in this calculation and potentially make it less reliable in some regimes: $\log \left(S / m^{2}\right)$ and $\log \left(p_{T}^{2} / m^{2}\right)$ become large when the center of mass energy $\sqrt{S}$ or the transverse momentum $p_{T}$ of the observed quark is much larger than its mass. Large $\log \left(1-4 m^{2} / \hat{s}\right)$ appear when the heavy quarks are produced close to the partonic threshold. I shall briefly describe the resummation of $\log (1-$

1) Review talk given at the b20 Symposium "Twenty Beautiful Years of Bottom Physics", Illinois Institute of Technology, Chicago, June 29 - July 2, 1997.

2) Present address: LPTHE, Université Paris-Sud, Orsay, France 
$\left.4 m^{2} / \hat{s}\right)$ and $\log \left(p_{T}^{2} / m^{2}\right)$ terms, and the inclusion of non-perturbative fragmentation effects.

I shall finally also briefly comment on the subject of heavy quarkonium production, where our understanding seems to have been greatly increased by a lot of recent theoretical activity.

\section{NLO CALCULATION}

The road to the NLO evaluation of heavy quark hadroproduction cross sections was paved by Collins, Soper and Sterman [5], who argued that the following factorization formula holds:

$$
d \sigma\left(H_{1} H_{2} \rightarrow Q \bar{Q} ; m\right)=\sum_{i j} \int f_{i / H_{1}} f_{j / H_{2}} d \hat{\sigma}(i j \rightarrow Q \bar{Q} ; m)+\mathcal{O}\left(\frac{\Lambda_{Q C D}}{m}\right),
$$

where the summation over partons $i$ and $j$ runs only over gluons and light quarks, and the heavy quarks are generated only at the perturbative level, by gluon splitting. The cross section explicitly depends on the heavy quark mass $m$ and on all other scales entering the problem (total energy $\sqrt{S}$, transverse momentum $p_{T}$, etc.).

Along these lines, explicit calculations were performed by two groups, Nason, Dawson and Ellis [6] on one side and Beenakker, Kuijf, Meng, van Neerven, Schuler and Smith [7] on the other. More recently Mangano, Nason, and Ridolfi (MNR) [8] have presented a Montecarlo integrator, based on the first of the two calculations, which can provide fully exclusive cross sections, thereby allowing detailed comparisons with experimental data. A very extensive collection of such comparisons is presented in a recent review [9], from which we select some plots to be shown here.

The ratios of next-to-leading over leading order predictions for total cross sections depend on $m / \sqrt{S}$ and are about 1.3 for top production at Tevatron energy $(\sqrt{S}=$ $1800 \mathrm{GeV}$ ) and of order 2 or larger for charm and bottom already at fixed target energy. Large uncertainties, due to monotonic renormalization/factorization scale dependence, are present for charm and bottom, while the prediction is fairly reliable for top $( \pm 10 \%)$, as shown in figure 1 .

One-particle inclusive differential cross sections, usually $p_{T}$ distributions, can also be considered. Fig. 2 shows on the left a comparison of E769 pion-nucleon fixed target data with NLO QCD plus two non-perturbative contributions: an intrinsic initial transverse momentum $k_{T}$ of the colliding partons, with $\left\langle k_{T}\right\rangle=1 \mathrm{GeV}$, and fragmentation effects of the charm into the observed charmed hadrons, described with the aid of a Peterson [10] fragmentation function (FF) with $\epsilon=0.06$. The overall result (the dot-dashed line) can be seen to be slightly softer than the data. Uncertainties are however large: a larger mass, a larger $\left\langle k_{T}\right\rangle$ or a harder $\mathrm{FF}$ could help improve the agreement. We should mention that, while the inclusion of the non-perturbative contributions might appear unnecessary here, it looks however mandatory when considering two-particle distributions or even $p_{T}$ distributions in 

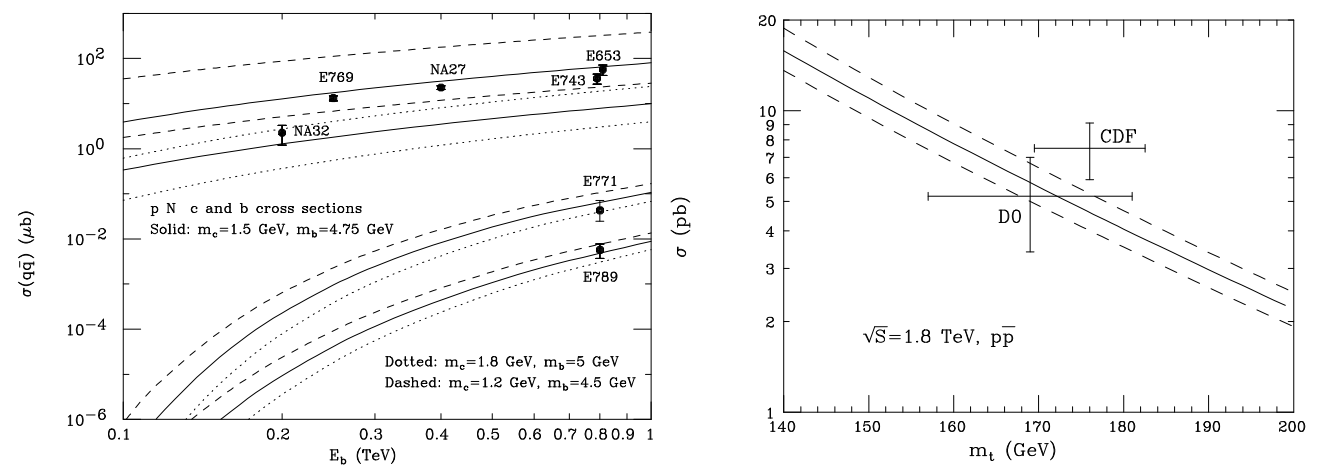

FIGURE 1. Charm and bottom production at fixed target experiments vs. NLO theoretical predictions (left), and top production at the Tevatron (right), from ref. [9].
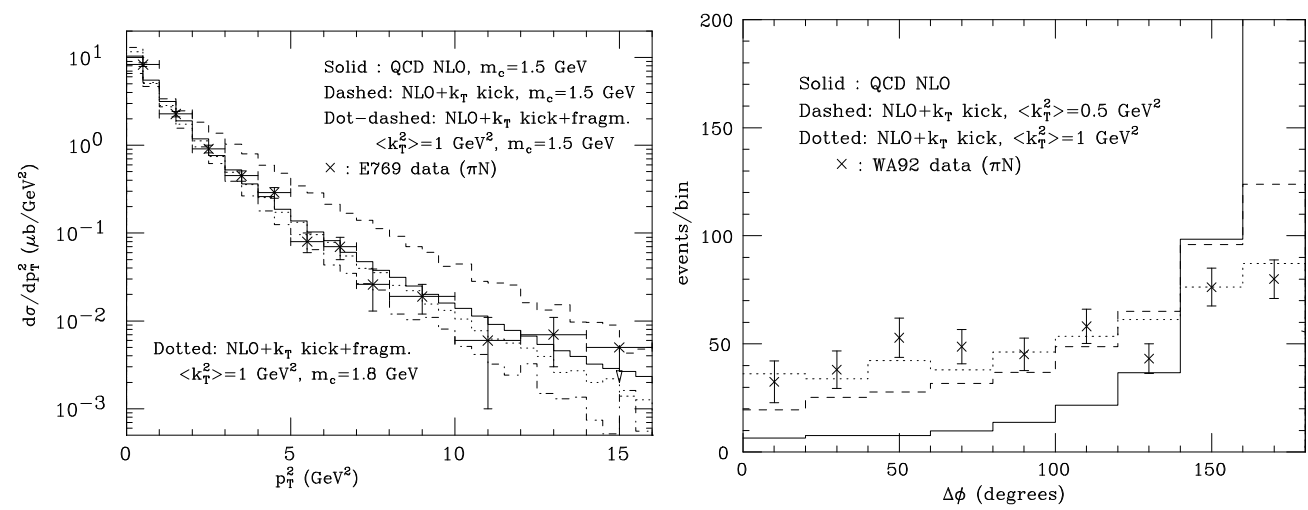

FIGURE 2. Charm $p_{T}$ inclusive distribution in $\pi N$ collisions vs. theoretical predictions [NLO calculation + initial $k_{T}\left(\left\langle k_{T}^{2}\right\rangle=1 \mathrm{GeV}^{2}\right)+$ non-pert. fragmentation (Peterson, $\left.\left.\epsilon=0.06\right)\right]$ on the left, and azimuthal correlation of the $c \bar{c}$ pair on the right. From ref. [9].

photon-hadron collisions, pure NLO QCD being there clearly unable to describe the data by itself. An example is given in fig. 2, on the right, where WA92 pion-nucleon data for the azimuthal distance $\Delta \phi$ of the $c$ and the $\bar{c}$ are compared to theoretical predictions: the inclusion of the $k_{T}$ kick brings the curve into agreement with the data. More such comparisons can be found in [9]. The overall picture suggests some consistency between the non-perturbative inputs needed to describe the oneand the two-particle distributions in both photo- and hadroproduction.

More comparisons of $p_{T}$ distributions can be done with the data for bottom production taken at the Tevatron by the CDF and D0 experiments. These distributions initially caused some concern, as they were markedly higher than the NLO QCD predictions. The data have now come down a bit, thanks to a better understanding of some decay chains used in the analysis (like the $B \rightarrow J / \psi \rightarrow \mu^{+} \mu^{-}$one) and to the use of microvertex silicon detectors which allow a much better identification and reconstruction of the heavy quark events. But, still, theory and data are not 

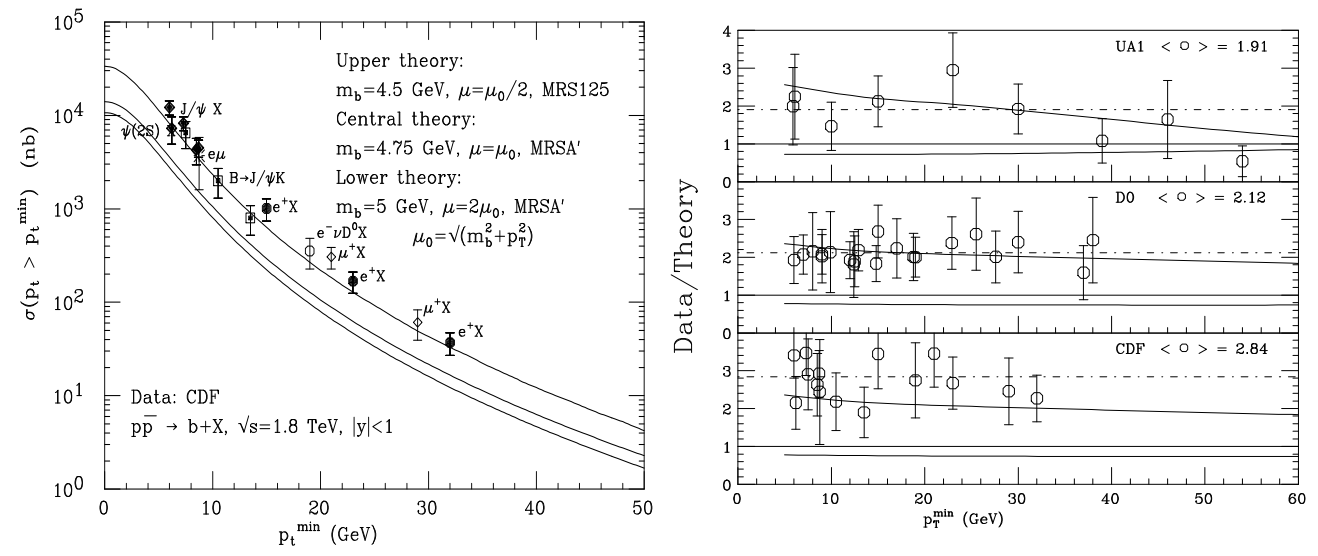

FIGURE 3. Bottom $p_{T}$ distributions data versus theoretical predictions, from ref. [9].

in perfect agreement. Fig. 3 shows on the left the data from CDF and on the right the data/theory ratios from CDF, D0 and UA1. Data can be seen to overshoot the central theoretical predictions by factors between two and three, and to be in fair agreement with the upper edge of the theoretical uncertainty band. One can thus conclude that no serious disagreement is present, but that the situation certainly deserves further investigations, also in the light of data on forward bottom production by the D0 Collaboration, which appear to be about a factor of four higher than the theoretical prediction.

\section{RESUMMATION OF THRESHOLD EFFECTS}

As we mentioned in the Introduction, potentially large logarithms appear in the NLO fixed-order calculation and make it less reliable in some regimes. We deal now with $\log \left(1-4 m^{2} / \hat{s}\right)$ terms, which appear in the partonic cross section in the form

$$
\hat{\sigma}(\hat{s})=\sigma_{0}(\hat{s})\left[1+C \alpha_{s} \log ^{2}\left(1-\frac{4 m^{2}}{\hat{s}}\right)+\cdots\right] .
$$

These logarithms become large when the cms energy the colliding partons have is close to the invariant mass of the two produced heavy quarks, that is to say, when the quarks are produced close to the threshold. Resummation of such terms can turn out to be phenomenologically important when the quark is very heavy with respect to the hadronic collision energy: top production at the Tevatron, with the top weighing $175 \mathrm{GeV}$ and the machine delivering $1800 \mathrm{GeV}$, could be such a case.

In order to better investigate this issue three groups have in recent years attempted such a resummation. We are not going to go into any details here, to be found in the respective papers, but simply summarize their conclusions and point to similarities and differences of approaches and results. 
Laenen, Smith and van Neerven [11] performed the resummation in $x$-space, by directly exponentiating the leading $\log (\mathrm{LL}) \alpha_{s} \log ^{2}(1-x)$ term, where $x=$ $4 \mathrm{~m}^{2} / \hat{s}$. Their formulae necessitate the introduction of an artificial infrared cutoff which serves a double purpose: it avoids hitting the Landau pole when the now $z$-dependent argument of the running coupling $\alpha_{s}$ tends to zero as $z \rightarrow 1$, and it

regulates an otherwise divergent integral of the form $\int_{0}^{1} d z \exp \left[|a| \log ^{2}(1-z)\right]$. They predict a $10 \%$ increase over the NLO fixed order calculation for top production at the Tevatron, but with a large uncertainty due to the dependence on the unphysical cutoff.

Berger and Contopanagos [12] instead perform the resummation in Mellin moments space, and then invert back to $x$-space for producing phenomenological predictions. When performing this inversion they apply a so-called principal value prescription to deform the integration contour and avoid hitting the Landau pole. They then discard all the non-leading terms generated by the inverse transform, arguing they are not universal, and only retain the LL ones. The further need for an infrared cutoff to avoid the $\int_{0}^{1} d z \exp \left[|a| \log ^{2}(1-z)\right]$ divergence is met by choosing it in such a way that all integrations are confined to a perturbative domain, i.e. to regions where the discarded non-leading terms are really subdominant. Through this procedure they also find an increase of about 10\%, but claim a small uncertainty due to the motivated choice of the cutoff.

A third evaluation of soft-gluon resummation effects has been performed by Catani, Mangano, Nason and Trentadue [13]. They also perform the resummation in Mellin space, and avoid the Landau pole by what they call minimal prescription, i.e. a choice of the integration contour which leaves the non-perturbative branch cut to the right. After doing so, they perform the $z$ integration by retaining both the leading and the next-to-leading (NLL) terms. Their argument is that the NLL contributions are generated by momentum conservation and, while formally subleading, discarding them leads to factorially divergent integrals and hence to the need for a cutoff. The phenomenological outcome of their investigation, at variance with previous findings, is that top hadroproduction at the Tevatron energy is predicted to increase by only $1 \%$ with respect to the fixed order result. They also claim little uncertainty on this result due to the absence of an explicit cutoff.

Whatever the approach one considers correct, we are however still far from experimentally probing such fine details (see experimental errors in fig. 1).

\section{LARGE TRANSVERSE MOMENTUM RESUMMATION}

Another kind of large logarithmic terms appearing in the NLO calculation and eventually spoiling its convergence $\operatorname{are} \log \left(p_{T}^{2} / m^{2}\right)$ terms. They also need therefore to be resummed to all orders to allow for a sensible phenomenological prediction. Such a resummation has been performed along the following lines [14]. 

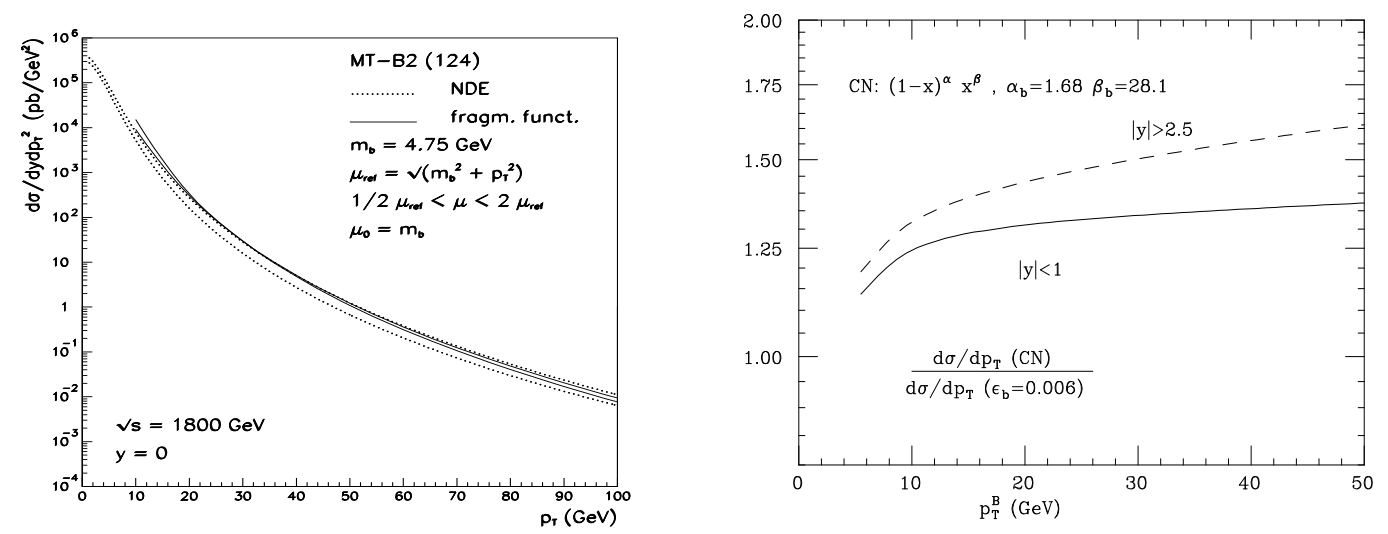

FIGURE 4. Comparison of bottom $p_{T}$ distributions in the fixed order (NDE) and resummed approaches (left plot, from ref. [14]), and effect of a harder $b \rightarrow B$ fragmentation function on bottom production at the Tevatron [18] (right plot).

One observes that in the large- $p_{T}$ limit $\left(p_{T} \gg m\right)$ the only important mass terms are those appearing in the logs, all the others being power suppressed. This means that an alternative description of heavy quark production can be achieved by considering massless quarks and providing at the same time perturbative distribution and fragmentation functions also for the heavy quark, describing the logarithmic mass dependence. The factorization formula becomes

$$
d \sigma\left(p_{T}\right)=\sum_{i j k} \int F_{i / H_{1}}(\mu,[m]) F_{j / H_{2}}(\mu,[m]) d \hat{\sigma}\left(i j \rightarrow k ; p_{T}, \mu\right) D_{k}^{Q}(\mu, m),
$$

with parton indices $i, j$ and $k$ also running on $Q$, taken massless in $\hat{\sigma}$, now an $\overline{\mathrm{MS}}$ subtracted cross section for light parton production. The dependence on $m$ of the parton distribution functions $F_{i / H}$, shown between square brackets in eq. (3), is only there when $i$ or $j$ happens to be the heavy quark $Q$.

The key point is that the large mass of the heavy quark allows for the evaluation in perturbative QCD (pQCD) of its distribution and fragmentation functions. Initial state conditions for $F_{Q / H}\left(\mu_{0}=m\right)$ [15] and $D_{k}^{Q}\left(\mu_{0} \simeq m\right)$ [16] can be calculated in pQCD at NLO level in the $\overline{\mathrm{MS}}$ scheme. These initial state conditions can then be evolved with the Altarelli-Parisi equations up to the large scale set by $\mu \simeq p_{T}$. This evolution will resum to all orders the large logarithms previously mentioned.

It is important to mention that due to the neglecting of power suppressed mass terms this approach becomes unreliable when $p_{T} \simeq m$. In this region only a case by case comparison with the full NLO massive calculation - here reliable and to be taken as a benchmark - can tell how accurate the resummed result is.

Phenomenological analyses [14] show that the effect of the resummation becomes sizeable only at very large $p_{T}$, say greater than $50 \mathrm{GeV}$ for bottom hadroproduction 
at the Tevatron, resulting in a milder factorization/renormalization scale dependence of the result and in a slightly softer $p_{T}$ spectrum.

Non-perturbative effects describing, say, the $b \rightarrow B$ meson transition can also be included within this formalism. The $b \rightarrow B$ fragmentation function can be fitted to LEP $e^{+} e^{-}$data and then used for predicting $B$ cross sections at the Tevatron. Recent analyses [17] show that this FF, when used in connection with a NLO evaluation of bottom production like the MNR one, should probably be taken harder than commonly done in the past. This choice of a harder non-perturbative FF would increase the Tevatron cross sections from twenty to fifty per cent [18] (see fig. 4), helping to reconcile theory and experimental data.

\section{HEAVY QUARKONIUM PRODUCTION}

The production of heavy quarkonia has been subjected to intense study in the last two or three years, with tens of papers having being produced on the problem of $J / \psi, \psi^{\prime}, \chi$ and $\Upsilon$ production in $e^{+} e^{-}, \gamma p, p \bar{p}, p N, \pi N$ collisions.

The reason for such a surge in interest was the appearance of a theoretical framework, the so called Factorization Approach (FA) by Bodwin, Braaten and Lepage [19], which seems able to solve the theoretical problems that quarkonium production models faced in the past, and also to reconcile theoretical predictions with experimental data, previously in disagreement up to factors of fifty in some instances.

In this talk I shall not review the Factorization Approach in detail, leaving this theoretical introduction to other sources (see for instance [20]). I shall just recall how the FA writes the quarkonium state $H$ production cross section in the following form:

$$
\sigma(i j \rightarrow Q \bar{Q} \rightarrow H)=\sum_{n} \hat{\sigma}(i j \rightarrow Q \bar{Q}[n])\left\langle\mathcal{O}^{H}(n)\right\rangle
$$

According to this equation, the cross section for producing the observable quarkonium state $H$ is factorized into two parts. In the short distance part a $Q \bar{Q}$ pair of heavy quarks is produced in the spin/colour/angular momentum state ${ }^{2 S+1} L_{J}^{(c)} \equiv n$ by the scattering of the two light partons $i$ and $j$. Subsequently this pair hadronizes into the quarkonium $H ;\left\langle\mathcal{O}^{H}(n)\right\rangle$ is formally a non-relativistic QCD (NRQCD) matrix element describing this non-perturbative transition.

An important feature of this equation is that also $Q \bar{Q}$ pairs in a colour octet state are allowed to contribute to the production of a colour singlet quarkonium $H$ : their colour is neutralized via a non-perturbative emission of soft gluons. While the corresponding matrix elements are suppressed by the need of such an emission, the short distance coefficients can on the other hand be large, perhaps overcompensating the suppression of the non-perturbative term. This explains why colour octet contributions can play a very important role in predicting the total size of quarkonium production cross sections. 
The limited space available doesn't allow any detail about the phenomenological studies which have been performed. Ref. [21] contains a small up-to-date review, with references to the original papers. I shall only mention here that the introduction of the colour octet channels allows for what looks like a "reasonable" description of the data. Colour octet matrix elements have to be fitted to the data themselves, and the uncertainties on these fits are certainly not smaller than a factor of two, due to the many systematics entering their determination: parton distribution functions, charm quark mass, $\alpha_{s}$ value, higher order QCD corrections, etc. However, the values one gets appear of the correct order of magnitude if compared via NRQCD scaling rules [22] to the colour singlet ones, thereby supporting the underlying picture.

So far all fits have been performed using leading order cross sections. Very recently a next-to-leading order calculation for quarkonium total cross sections, both via singlet and octet channels, has been completed [23]. It will therefore be possible to reduce at least some of the uncertainties previously mentioned and hence obtain more reliable fits.

\section{REFERENCES}

1. J.J. Aubert et al., Phys. Rev. Lett. 33, 1404 (1974);

J.-E. Augustin et al., Phys. Rev. Lett. 33, 1406 (1974).

2. T. Appelquist and H.D. Politzer, Phys. Rev. Lett. 34, 43 (1975);

A. De Rujula and S.L. Glashow, Phys. Rev. Lett. 34, 46 (1975);

C.A. Dominguez and M. Greco, Lett. Nuovo Cim. 12, 439 (1975).

3. S.W. Herb et al., Phys. Rev. Lett. 39, 252 (1977).

4. F. Abe et al. (CDF Coll.), Phys. Rev. D50, 2966 (1994);

F. Abe et al. (CDF Coll.), Phys. Rev. Lett. 74, 2626 (1995);

S. Abachi et al. (D0 Coll.), Phys. Rev. Lett. 74, 2632 (1995).

5. J.C. Collins, D. Soper and G. Sterman, Nucl. Phys. B263, 37 (1986).

6. P. Nason, S. Dawson and R.K. Ellis, Nucl. Phys. B303, 607 (1988);

P. Nason, S. Dawson and R.K. Ellis, Nucl. Phys. B327, 49 (1989), erratum ibid. B335 (1990) 260.

7. W. Beenakker, H. Kuijf, W.L. van Neerven and J. Smith, Phys. Rev. D40, 54 (1989); W. Beenakker, W.L. van Neerven R. Meng, G.A. Schuler and J. Smith, Nucl. Phys. B351, 507 (1991).

8. M.L. Mangano, P. Nason and G. Ridolfi, Nucl. Phys. B373, 295 (1992).

9. S. Frixione, M.L. Mangano, P. Nason and G. Ridolfi, CERN-TH/97-16 (hep$\mathrm{ph} / 9702287)$.

10. C. Peterson, D. Schlatter, I. Schmitt and P.M. Zerwas, Phys. Rev. D27, 105 (1983).

11. E. Laenen, J. Smith and W.L. van Neerven, Nucl. Phys. B369, 543 (1992);

E. Laenen, J. Smith and W.L. van Neerven, Phys. Lett. B321, 254 (1994).

12. E. Berger and H. Contopanagos, Phys. Lett. B361, 115 (1995);

E. Berger and H. Contopanagos, Phys. Rev. D54, 3085 (1996). 
13. S. Catani, M.L. Mangano, P. Nason and L. Trentadue, Phys. Lett. B378, 329 (1996);

S. Catani, M.L. Mangano, P. Nason and L. Trentadue, Nucl. Phys. B478, 273 (1996).

14. M. Cacciari, M. Greco, Nucl. Phys. B421, 530 (1994).

15. J.C. Collins and W.-K. Tung, Nucl. Phys. B278, 934 (1986).

16. B. Mele and P. Nason, Nucl. Phys. B361, 626 (1991).

17. G. Colangelo and P. Nason, Phys. Lett. B285, 167 (1992);

M. Cacciari and M. Greco, Phys. Rev. D55, 7134 (1997).

18. M.L. Mangano, private communication.

19. G.T. Bodwin, E. Braaten and G.P. Lepage, Phys. Rev. D51, 1125 (1995), erratum ibid. D55 (1997) 5853.

20. E. Braaten, S. Fleming and T.C. Yuan, Ann. Rev. Nucl. Part. Sci. 46, 197 (1996);

E. Braaten, Talk given at 3rd International Workshop on Particle Physics Phenomenology, Taipei, Taiwan, 14-17 Nov 1996, OHSTPY-HEP-T-97-004 (hep$\mathrm{ph} / 9702225)$;

M. Beneke, Lecture at the XXXIV SLAC Summer Institute on Particle Physics (August 1996), CERN-TH/97-55 (hep-ph/9703429).

21. M. Cacciari, Proceedings of the XXXII Rencontres de Moriond "QCD and Hadronic Interactions", Les Arcs, March 1997, DESY 97-091 (hep-ph/9706374).

22. G.P. Lepage, L. Magnea, C. Nakhleh, U. Magnea, and K. Hornbostel, Phys. Rev. D46, 4052 (1992).

23. A. Petrelli, M. Cacciari, M. Greco, F. Maltoni and M.L. Mangano, CERN-TH/97142 (hep-ph/9707223). 